\title{
A coupled transport model for water splitting within a porous metal oxide thermochemical reactor using the random walk particle tracking method
}

\author{
Nima Rahmatian ${ }^{\mathrm{a}, 1}$, Renwei Mei ${ }^{\mathrm{a}}$, James Klausner ${ }^{\mathrm{a}}$, Jörg Petrasch ${ }^{\mathrm{b}}$ \\ ${ }^{a}$ Department Of Mechanical and Aerospace Engineering, University of Florida, Gainesville, FL, USA \\ ${ }^{\mathrm{b}}$ Energy Research Center, Vorarlberg University of Applied Sciences, 6850 Dornbirn, Austria
}

\begin{abstract}
Water splitting using an iron-based looping processes is a promising method to produce high purity hydrogen. The cyclical, heterogeneous oxidation and reduction reactions are carried out within high surface area stable porous structures as the solid reactant. The random walk method is used to simulate chemical reaction and species transport, as it is capable of handling stiff reaction kinetics and varying hydrodynamic dispersion tensors caused by pore-level velocity fluctuations. The original random walk formulation is extended to account for bulk density variations and source terms due to the chemical reaction. The species transport equation is recast in the form of the Fokker-Planck equation, and the trajectories of fluid particles are obtained by solving an appropriate Langevin equation that has additional drift terms as a result of spatial variations in bulk density and dispersion tensor. The source term is accounted for by changing the number or the composition of fluid particles based on the reaction kinetics. The extended approach for each new term is validated against exact solutions or highly resolved finite difference solutions. Finally, a new coupled model for bulk fluid flow, species transport, and chemical reaction in porous media is developed and applied to simulate a bench-scale water splitting reactor with a porous iron-silica fixed bed structure. Excellent agreement with the measured hydrogen production rate at different operating conditions is obtained.
\end{abstract}

Keywords: Random Walk, Magnetically Stabilized Porous Structure, Water-Splitting, Metal/Metal Oxide Looping Process, Species Transport, Coupled Model.

\section{Introduction}

Clean and renewable sources of energy are critical for long term human development. Hydrogen produced from sustainable resources is an ecologically-benign fuel which can be used in electrochemical cells as well as for direct combustion. There are numerous processes that generate hydrogen from water, fossil and renewable biomass resources. These processes include reforming and pyrolysis as well as electrolysis and thermolysis [1-4]. Currently, the leading technology for producing hydrogen in large quantities is steam reforming of methane [5]. Solar

\footnotetext{
${ }^{1}$ Corresponding author, Department of Mechanical and Aerospace Engineering, University of Florida, Gainesville, FL 32611, USA, P.O. Box 116250. Tel: +1 321947 7442, Fax:+1 352392 1071, Email: nrah@ufl.edu
} 
thermal chemical cycles, especially cycles involving metal/metal oxide systems, have recently drawn a lot of attention[6,7]. In these processes, hydrogen is produced from water by oxidizing a metal, and the subsequent metal oxide is reduced back to metal using solar heat in a thermal reduction step $[8,9]$.

Mehdizadeh et al. [10] reported a novel strategy for making magnetically stabilized porous structures to produce hydrogen through the looping process. This process involves the production of hydrogen from steam at high temperatures $\left(800{ }^{\circ} \mathrm{C}\right)$ by oxidizing the iron in the porous structure and reducing the iron oxide to elemental iron by flowing a reducing agent such as carbon monoxide, thus completing one cycle of the looping process (see Eq. 1). Carbon monoxide required for the reduction step is obtained from gasification of coal and therefore the energy content of coal is transferred to hydrogen with high purity which can be utilized as a clean fuel.

$3 \mathrm{Fe}+4 \mathrm{H}_{2} \mathrm{O} \rightarrow \mathrm{Fe}_{3} \mathrm{O}_{4}+4 \mathrm{H}_{2}$

$\mathrm{Fe}_{3} \mathrm{O}_{4}+4 \mathrm{CO} \rightarrow 3 \mathrm{Fe}+4 \mathrm{CO}_{2}$

This process involves mass, momentum, and species transport in porous media with significant chemical reactions between the solid and the gas phase. A detailed analysis or simulation that can account for those complex physical and chemical mechanisms is highly desirable in order to develop a better understanding of the process and provide useful guidelines for optimal operating conditions and engineering design.

Simulating the reaction of steam within a porous structure can be performed at the pore level in which the exact geometry of the porous media is used and fluid flow is simulated within the pore spaces. This method requires a high resolution representation of the porous media. Moreover the method is computationally expensive since all relevant small pore-level scales must be resolved. As an alternative, continuum models are developed by treating solid and fluid phases as interpenetrating continua using the method of volume averaging [11]. The computational cost of solving continuum models is significantly lower. However, effective transport properties of the solid matrix are required.

Species transport in porous media consists of macroscopic advection due to bulk fluid flow, dispersion due to irregular flow patterns caused by the presence of the solid matrix, and diffusion due to molecular motion. For advection dominated flows, the commonly used finite volume or finite difference methods for solving transport problems using an Eulerian formulation suffer from numerical dispersion and artificial oscillations. They require highly resolved grids and small time steps to overcome the associated errors $[12,13]$. The shortcomings of these methods are more pronounced when applied to heterogeneous porous media with small scale features [12]. 
Lagrangian methods, on the other hand, inherently have zero numerical dispersion and are suitable for heterogeneous porous media [12,14]. Typically computational costs of particle methods are relatively higher than those of Eulerian methods, but they are easy to parallelize and consequently computational time can be reduced significantly [15].

Due to unique features of the Lagrangian particle based methods, they are widely used in modeling solute transport within porous media. Ground water and contaminant flow simulations are essentially performed on large heterogeneous domains that involve several length scales and hence stochastic particle tracking methods, such as random walk (RW), are very well suited to obtain accurate results with high stability and efficiency. As a result, the majority of studies reported in the literature considers the reaction and transport of species within the liquid inside the porous underground reservoir [15-17]. Moreover, similar Lagrangian methods are developed and employed to simulate turbulent flows and solve the reaction-diffusion equations for propagating laminar flames[18-22].

In the present paper, the random walk particle method is used to simulate water-splitting processes within porous iron-silica structures. The coupling of bulk fluid flow and mass transport in this process requires modification to the conventional random walk particle method. The extended method with such modifications is validated against known exact solutions for special cases or high resolution finite difference solutions when no exact solution is available. The model is then used to predict hydrogen production rates within a laboratory scale reactor at different operating conditions, and results are compared with experimental measurements.

\section{Mathematical Model}

The volume averaging method is employed to develop the continuum scale model. The intrinsic volume average of a quantity $\phi$ is defined as [11] :

$$
\langle\phi\rangle^{F}=\frac{1}{V_{F}} \int_{V_{F}} \phi d V
$$

where $V_{F}$ is the volume filled with the fluid phase of a representative elementary volume (REV) [23] of the porous medium. The superficial average is the average over the total volume of all phases within a porous medium:

$$
\langle\phi\rangle=\frac{1}{V} \int_{V} \phi d V
$$

With the definition of porosity, $\varepsilon=\frac{V_{F}}{V}$, and the assumption of no macro-scale variation of $\varepsilon$, the intrinsic and superficial averages are related via: 


$$
\langle\phi\rangle=\varepsilon\langle\phi\rangle^{F}
$$

The volume averaged mass conservation equation for the fluid phase within porous media can be written as:

$$
\frac{\partial\left(\varepsilon\langle\rho\rangle^{F}\right)}{\partial t}+\nabla \cdot\left(\langle\rho\rangle^{F}\langle\mathbf{u}\rangle\right)=\langle\dot{r}\rangle
$$

where $\langle\rho\rangle^{F}$ is the averaged fluid mixture density, $\langle\mathbf{u}\rangle$ is the superficial average of the velocity vector, and $\langle\dot{r}\rangle$ is the net superficial average rate of volumetric mass production or destruction.

The species conservation equation can be similarly written considering both advection and diffusion fluxes of species to a control volume [24]:

$$
\frac{\partial}{\partial t}\left(\varepsilon\left\langle\rho_{j}\right\rangle^{F}\right)+\nabla \cdot\left(\langle\mathbf{u}\rangle\left\langle\rho_{j}\right\rangle^{F}-\langle\rho\rangle^{F} \mathbf{D} \cdot \nabla\left(\frac{\left\langle\rho_{j}\right\rangle^{F}}{\langle\rho\rangle^{F}}\right)\right)=\left\langle\dot{r}_{j}\right\rangle
$$

where $\left\langle\rho_{j}\right\rangle^{F}$ is the intrinsic average density of species $\mathrm{j}$; $\mathbf{D}$ is the hydrodynamic dispersion tensor defined as [15]:

$\mathbf{D}=\left(\alpha_{T}\left|\langle\mathbf{u}\rangle^{F}\right|+D_{m}\right) \mathbf{I}+\left(\alpha_{L}-\alpha_{T}\right) \frac{\langle\mathbf{u}\rangle^{F}\left(\langle\mathbf{u}\rangle^{F}\right)^{T}}{\left|\langle\mathbf{u}\rangle^{F}\right|}$

where $\alpha_{L}$ and $\alpha_{T}$ are dispersivity coefficients in the longitudinal and transverse directions within the porous media, respectively, and $D_{m}$ is the molecular diffusivity. For constant $\varepsilon$, Eq. 6 can be written as:

$\frac{\partial\left\langle\rho_{j}\right\rangle^{F}}{\partial t}+\nabla \cdot\left(\frac{\langle\mathbf{u}\rangle}{\varepsilon}\left\langle\rho_{j}\right\rangle^{F}\right)-\frac{1}{\varepsilon} \nabla \cdot\left(\langle\rho\rangle^{F} \mathbf{D} \cdot \nabla\left(\frac{\left\langle\rho_{j}\right\rangle^{F}}{\langle\rho\rangle^{F}}\right)\right)-\frac{1}{\varepsilon}\left\langle\dot{r}_{j}\right\rangle=0$,

The fluid momentum transport inside porous media is modeled based on Darcy's law:

$$
\langle\mathbf{u}\rangle=-\frac{K}{\mu} \nabla\langle p\rangle^{F}
$$


where $K$ is the permeability of the porous structure, $\mu$ is the fluid viscosity, and $\langle p\rangle^{F}$ is the intrinsic average of pressure.

Figure 1. The schematic of the random walk motion of particles in porous media and their mass and composition change due to chemical reaction

\section{Numerical Method}

The entire computational procedure starts with the species transport equation by introducing the fluid particles which are packets of mass consisting of all species and travel inside the porous medium as shown schematically in Fig. 1. The particles are moved based on the random walk model that has advective and diffusive steps [12,16,17,25]:

$$
\mathbf{x}_{p}^{n+1}=\mathbf{x}_{p}^{n}+\mathbf{A}\left(\mathbf{x}_{p}^{n}\right) \Delta t+\mathbf{B}\left(\mathbf{x}_{p}^{n}\right) \cdot \mathbf{z} \sqrt{\Delta t}
$$

Here $\mathbf{x}_{p}^{n}$ is the position of fluid particle at $t=n \Delta t, \mathbf{A}$ is drift vector, $\mathbf{B}$ is a diffusion matrix, and $\mathbf{z}$ is a vector of independent random numbers with zero mean and unit variance. For a large number of particles the probability density function, $f(\mathbf{x}, \mathrm{t})$, of finding particles at $\mathbf{x}$ satisfies the Fokker-Planck equation [26]:

$$
\frac{\partial f}{\partial t}+\nabla \cdot(\mathbf{A} f)-\nabla \nabla:\left(\frac{1}{2} \mathbf{B} \cdot \mathbf{B}^{\mathrm{T}} f\right)=0
$$

To recast the convection and diffusion terms of species transport equation (Eq. 8) in the form of Eq. 11, the drift vector and diffusion matrix need to be:

$$
\mathbf{A}=\frac{1}{\varepsilon}\left(\langle\mathbf{u}\rangle+\nabla \cdot \mathbf{D}+\frac{\mathbf{D}}{\langle\rho\rangle^{F}} \cdot \nabla\langle\rho\rangle^{F}\right)
$$

$$
\mathbf{B} \cdot \mathbf{B}^{\mathrm{T}}=2 \mathbf{D}
$$

Although in earlier works reported in the literature the effect of variable dispersion tensors has been neglected [27,28], it has been included in more recent studies [25,29]. However, by deriving the species transport equation from mass conservation for a fluid mixture, the bulk fluid density and its gradient now appears in the drift vector in order to recover the correct species transport. In the presence of chemical reactions, there are significant bulk density variations. For example, the mixture density can decrease by a factor of eight during full conversion of steam to 
hydrogen with water-splitting reactions inside the reactor [10]. Thus the density gradient will strongly affect the advective step of the fluid particles.

A source (or sink) for species transport can be modeled by changing the composition and mass of the particles according to the rate law of the chemical reaction, as opposed to generating and destroying particles. This is due to the fact that each fluid particle is composed of multiple species and molecular diffusion of species is neglected. Therefore, the simultaneous reactions between multiple species can be easily accounted for without the need to add separate particles for each species.

The random walk method has first order temporal accuracy for solving the Fokker-Planck $[30,31]$ equation, and statistical error is inversely proportional to $\sqrt{N_{p}}$, where $N_{p}$ is the number of fluid particles in the cell [32]. For the present work, there are strong variations of advective velocity due to variable density and mass generation due to chemical reactions. The impact of such factors on the accuracy of the random walk method is unknown.

In section 5, the modified random walk method with strong variations of advection and source (or sink) is validated by comparing with exact solutions of known cases for a uniform medium porosity.

\section{Coupled random walk model for the water-splitting reaction}

In order to simulate the flow of reacting gases in the porous media, the fluid flow mass and momentum conservation equations must be solved simultaneously together with the species transport equation with a specified chemical reaction rate law. For the present study, the temperature of the water splitting experimental system is maintained constant through active control, and thus isothermal analysis is sufficient; no heat transport modeling is required. A coupled model consisting of three modules is developed to solve the desired transport equations. A schematic of the individual modules and their connections in the coupled model is shown in Fig. 2.

Figure 2. Schematic depiction of the coupled model and their connections

The mass conservation (Eq. 5) is coupled with Darcy's law (Eq. 9). Ideal gas behavior is assumed. For the fluid mixture, a partial differential equation is obtained for the pressure,

$$
\left(\frac{1}{R T}\right) \frac{\partial\langle p\rangle^{F}}{\partial t}+\frac{1}{\varepsilon} \nabla \cdot\left(-\frac{K}{\mu_{F}}\langle\rho\rangle^{F} \nabla\langle p\rangle^{F}\right)=\frac{1}{\varepsilon}\langle\dot{r}\rangle
$$


where $R$ and $\mu_{F}$ are the respective gas constant and viscosity for the fluid mixture, and $T$ is the fluid temperature. The porosity and permeability of the iron-silica porous reactive structure used in this study were experimentally measured to be 0.63 and $8.14 \mathrm{E}-11 \mathrm{~m}^{2}$, respectively.

Equation 14 is solved using a finite volume scheme on a uniform grid. The boundary conditions are the specified mass flux at the reactor inlet and pressure at the outlet, which correspond to the experimental conditions within the hydrogen production reactor [33].

The chemical reaction rate for both oxidation and reduction steps are written in the form of an Arrhenius rate law with a function $f$ that accounts for the reaction type of the solid phase [33],

$$
\dot{r}_{H_{2}}=k_{0} e^{-\frac{E_{a}}{\bar{R} T}} \rho_{H_{2} O} f(x)
$$

where $k_{0}$ is the pre-exponent term, $E_{a}$ is the activation energy, $f$ is based on a hybrid model for surface and diffusion limited reaction regimes and is written as [33],

$$
f(x)=\left(\frac{a(1-x)^{2 / 3}}{\sqrt{\left(1-(1-x)^{1 / 3}\right)^{2}+a^{2}}}\right)
$$

Here $x$ is the extent of the reaction, and $a$ is an adjustable parameter to account for the weighting of the relative contribution of surface and diffusion reaction mechanisms. More details about reaction rate modeling and experimental procedures for determining $k_{0}$ and $E_{a}$ can be found in [33].

Figure 3. Schematic of the 1D computational domain for water-splitting reaction

At each time step, the pressure is solved first. The velocity field is then obtained based on Darcy's law (Eq. 9). The fluid particles are then moved based on the random walk scheme since both the drift velocity and dispersion tensor can be obtained with knowledge of the flow field and mixture density. The chemical reaction is computed for each fluid particle by integrating Eq. 15 with respect to time. The vector $\mathbf{n}_{p}$, which stores the content of each species present in a fluid particle, is updated as,

$$
\mathbf{n}_{p}=\left(\begin{array}{c}
n_{H_{2}}^{n+1} \\
n_{H_{2} O}^{n+1}
\end{array}\right)=\left(\begin{array}{c}
n_{H_{2}}^{n}+V_{p}\left(\dot{r}_{H_{2}}^{n} d t\right) / M_{H_{2}} \\
n_{H_{2} O}^{n}+V_{p}\left(\dot{r}_{H_{2} O}^{n} d t\right) / M_{H_{2} O}
\end{array}\right)
$$


where the superscripts denote the time step, $V_{p}$ is the fluid particle volume that is computed using the ideal gas equation of state, and $M_{\mathrm{H}_{2}}$ and $M_{\mathrm{H}_{2} \mathrm{O}}$ are the molar mass of hydrogen and water, respectively. The volumetric mass generation in Eq. 14 is obtained by adding net mass changes in all particles present in the control volume and dividing by the sum of their volumes $V_{p}$. Next, the macroscopic properties, such as species density, mixture density, viscosity, and gas constant are obtained by averaging over the particles in each control volume. The new time step restarts by solving the pressure and velocity fields.

\section{Validation of modified random walk method for species transport equation}

Classical random walk is capable of solving the Fokker-Planck equation (Eq.11) accurately and efficiently. However, recasting the species transport equation (Eq. 8) in the form of Eq. 11 introduces new terms in the drift velocity (Eq. 12) due to variable $\langle\rho\rangle^{F}$ and $D$. Additionally, the source or sink of species is not directly accounted for in Eq. 11. In order to ensure that the random walk particle tracking method can be extended to study water-splitting reactions within porous media, several test cases, having exact solutions or highly resolved finite difference solutions, are selected for validation. All cases involve solving the advection diffusion (AD) equation subjected to different flow conditions and chemical reactions. A list of the validation cases are given in Table 1.

Table 1. List of validation tests

\subsection{CASE 1: One-D advection and diffusion of species for an instantaneous point source}

First, the advection and diffusion of species within an infinite domain with constant $u$ and $D$ is studied. An instantaneous point source of species $\mathrm{j}$ is simulated by specifying the initial species density of $\rho_{j}(x, t=0)=\delta(x)$ where $\delta$ is the Dirac delta function. The fluid bulk density is constant and there is no generation of species in the domain. Hence, Eq. 8 reduces to an Advection Diffusion equation with constant coefficients and can be written as:

$$
\frac{\partial \rho_{j}}{\partial t}+u \frac{\partial \rho_{j}}{\partial t}-D \frac{\partial^{2} \rho_{j}}{\partial x^{2}}=0
$$

Fluid particles consisting of only species $j$ are generated at $\mathrm{x}=0$ and tracked based on Eq. 10. The density distributions obtained using the random walk method are compared with the solution given by: 


$$
\rho_{j}(x, t)=\frac{M_{j}^{*}}{\sqrt{4 \pi D\left(t-t_{0}\right)}} \exp \left[-\frac{\left(x-x_{0}-u\left(t-t_{0}\right)\right)^{2}}{4 D\left(t-t_{0}\right)}\right]
$$

where $M_{j}^{*}=$ mass of species $j$ introduced initially at the point source. In Fig. $4, \rho_{j}^{*}=\frac{\rho_{j}}{M_{j}^{*} / d x}$ is shown for $\mathrm{Pe}=u L / D$ numbers of 10,50 , and 100 at different instances in time. A random walk simulation is performed using 50,000 particles to represent initial mass of species $j$ introduced in the bulk fluid stream. The figures are shown at different non-dimensional times $t^{*}=t u / L$.

Figure 4. Comparison for the normalized density of contaminants with the exact solution for $\mathrm{Pe}=10,50$, and 100 .

The simulation results are in excellent agreement with the exact solution, and no numerical diffusion is observed, even for high Pe numbers. This demonstrates that the basic steps of the random walk particle tracking method have been implemented correctly.

\subsection{CASE 2: Variable fluid bulk density}

For the oxidation and reduction reactions considered, the bulk fluid density changes due to chemical reactions. Thus a variable bulk density $\rho$ is used to mimic the significant change in mixture density in the reactor and to investigate its effect on the accuracy of the solution for species density. The validation is accomplished by comparing the random walk results with the highly resolved finite difference solution.

The transport of species $j$ inside a spatially variable fluid mixture is simulated. The reactor is assumed to have zero concentration of species $j$ initially. The species density is specified to be 1 at the inlet. The corresponding fluid particles are generated at inlet and moved using the RW scheme. The advection velocity varies corresponding to changes in fluid bulk density so as to conserve mass, while the effect of species transport on the flow field is neglected. The dispersion coefficient is set to be constant and Pe number is 0.1 .

The variable bulk density is assumed to have the following form (see Fig. 5)

$\rho^{*}(x, t)=\frac{\rho(x, t)}{\rho_{0}}=(1-\gamma)\left(1-x^{*}\right)^{3}+\gamma$

where $\rho_{0}$ is the inlet bulk density and $\gamma$ is the relative change in bulk density from inlet to outlet. Thus, the flow velocity normalized by inlet velocity $u_{0}$ is found to be: 


$$
u^{*}(x, t)=\frac{u(x, t)}{u_{0}}=\frac{1}{(1-r)\left(1-x^{*}\right)^{3}+r}
$$

In this study $\mathrm{r}$ is set to be $1 / 9$ corresponding to the change of density in oxidation reaction (Eq. 1a) assuming full conversion of steam to hydrogen.

Figure 5 shows profiles of $\rho_{j}^{*}$ at three instances. The finite difference (FD) solution is obtained on the discretized domain of $n_{\mathrm{x}}=1024$ intervals to ensure high accuracy. The time steps are 0.001 and 0.0001 seconds for FD and RW, respectively. Good agreement between the results from the two methods is achieved. It is concluded that the effect of significant change in bulk density can be accounted for by adding its gradient to the drift velocity in RW formulation (see Eq. 12).

Figure 5. Comparison of the species density profile with that predicted using finite difference at three time instances.

\subsection{CASE 3: Variable bulk fluid velocity $u$ and diffusion coefficient D}

In case 3 the transport of species is simulated under varying velocity and diffusion tensor (the bulk density is set to 1.0). The velocity is assumed to be linearly increasing in space and the diffusion coefficient is a second order polynomial of the local velocity which reflects reality over a wide range of conditions for transport problems [34].

The species equation thus reduces to

$\frac{\partial \rho_{j}}{\partial t}+\frac{\partial}{\partial x}\left(u \rho_{j}\right)-\frac{\partial}{\partial x}\left(D \frac{\partial}{\partial x}\left(\rho_{j}\right)\right)=0$

where

$\left\{\begin{array}{c}u(x, t)=u_{0}(1+a x) \\ D(x, t)=D_{0}(1+a x)^{2}, \quad a=b / L\end{array}\right.$

The boundary conditions are specified as,

$\rho_{j}^{*}=\rho_{j} / \rho_{j 0}=1$

$\left.\frac{\partial \rho_{j}}{\partial x}\right|_{x=L}=0$

The exact solution for this configuration is given in Kumar et al. [34]. The flow is simulated by generating fluid particles at the inlet and moving them following the random walk equation (Eq. 10). The effect of variable dispersion coefficient $D$ is accounted for in the drift velocity $\boldsymbol{A}$. The 
results of the simulation for $\mathrm{b}=0.1,0.5$, and 2.0 are shown at $t^{*}=t u_{0} / L=0.25$ and 0.75 in Fig. 6 . A total of 8,000 particles per cell are used at the inlet. Excellent agreement with the analytical solution [34] is observed.

Figure 6. Comparison of species density profile with that predicted using finite difference at two time instances for variable $u$ and $D$ with $b=0.1,0.5$, and 2.0. $\left(\mathrm{u}_{0}=0.05, \mathrm{D}=0.001, \mathrm{Pe}=50\right)$

\subsection{CASE 4: Source term with constant $u$ and D}

As stated previously the source or sink term is treated by changing the amount of species within each fluid particle in the random walk method. Thus for chemical reactions with the product of species $j$, the amount (mass) of species $j$ in fluid particles subjected to reaction increases, which leads to an increase in average species density. The validation of this approach is conducted through two test cases: 1) constant uniform generation $\left(\dot{r}_{j}=C\right)$; and 2) source term based on first order reaction kinetics $\left(\dot{r}_{j}=C \rho_{j}\right)$. In both cases the advection velocity and dispersion coefficient are assumed to be constant $\left(u=0.01 \mathrm{~m} / \mathrm{s}, D=0.001 \mathrm{~m}^{2} / \mathrm{s}\right)$ and the fluid mixture density is not affected by the species $j$.

\subsubsection{Constant source term}

The variation of species density from both exact [35] and random walk solutions is shown in Fig. 7. The inlet density is maintained constant. The reactor is assumed to have $\rho_{j}=0$ initially and $\left\langle\dot{r}_{j}\right\rangle$ is set to $0.05 \mathrm{~kg} /\left[\mathrm{m}^{3} \mathrm{~s}\right]$. The number of fluid particles in each cell is approximately 10,000 .

The results from the RW simulation agree well with the exact solution. It is noted that uniform generation causes a uniform increase in species density in the region $x>u t$ where the influence of the inlet condition by advection has not been reached.

Figure 7. Comparison with species density profile with the exact solution for constant generation

Figure 8. Comparison of species density profile with exact solution for first order generation

\subsubsection{Generation with first order chemical reaction}

Assuming a first order chemical reaction, the generation term is then linearly proportional to the species density. The initial and boundary conditions are similar to the uniform generation case. The comparison between the RW and exact solution [35] for the species density are shown in Fig. 8. The evolution of specie density, $\rho_{j}^{*}$, is accurately captured by the modified random walk method. 


\section{Application to simulating water-splitting process}

The efficacy of hydrogen production is characterized by the measured flow rate of hydrogen at the outlet of the reactor. This corresponds to the sum of the hydrogen content of fluid particles that leave the reactor. The effects of temperature, inlet flow rate of steam, and inlet concentration of steam on the hydrogen production rate are studied. The predicted hydrogen production from the coupled model is compared to experimental data from a cylindrical reactor reported in [33]. The detailed description of the experimental facility has been reported in Mehdizadeh et al. [10]. Due to uniformity of the flow field in radial and azimuthal directions in the experiment, all the variables are averaged over the cross section.

The predicted hydrogen production for the oxidation step at 800,700 and $600^{\circ} \mathrm{C}$ are shown in Fig. 9 together with the measured data, and very good agreement is observed. In this case, the velocity field and consequently dispersion tensor vary with time and space due to the chemical reaction. The change in species results in variable density, viscosity, and gas constant. The modified random walk method is able to successfully account for these variations in mixture properties and accurately predict the production of reactor products.

In order to analyze the performance of the reactor and optimize the operating conditions, the knowledge of the velocity profile, pressure, and bulk density distributions within the reactor is essential. Since the experimental measurements for these quantities are practically difficult or impossible, the coupled model can serve as a useful tool to obtain the desired quantities inside the reactor. Figure 10 shows pressure distribution as well as the mixture density profile inside the reactor. The decrease in the pressure is mainly due to the friction between the fluid and the solid porous bed.

Figure 9. Comparison of the predicted hydrogen production rate at the reactor outlet with experimental measurements for 600,700 , and $800^{\circ} \mathrm{C}$ and $3 \mathrm{~g} / \mathrm{min}$ steam mass flow rate.

Figure 10. Pressure and bulk density profiles along the reactor centerline for oxidation at $800^{\circ} \mathrm{C}$

Figure 11. Average axial velocity along the reactor for oxidation at $800^{\circ} \mathrm{C}$

Because the number of moles of the gas phase reactants is the same as that of the products during each reaction, the volumetric flow rate of fluid changes only slightly due to density variation and the effective viscosity of the mixture. Thus, as depicted in Fig. 11, the change in the magnitude of the velocity remains less than 5 percent. Since the fluid velocity is obtained by differentiating the pressure field according to Darcy's law, the statistical noise of the particle tracking method is more apparent in the velocity profile shown in Fig.11. 
Figure 12 shows molar fractions of $\mathrm{H}_{2}$ and $\mathrm{H}_{2} \mathrm{O}$ in the reactor at different instants of the oxidation step at $800^{\circ} \mathrm{C}$. Initially almost 60 percent of the steam reacts with the solid. As the iron in the structure oxidizes this conversion rate reduces until the hydrogen outflow is negligible and almost entirely steam exits unreacted. As a matter of practical operation of the reactor, the inlet steam flow rate can be adjusted based on the available iron in the structure; operating with variable steam flow rate will avoid an excessive amount of wasted energy in the conversion of water to steam.

Fig. 12 Transient variation in species molar fractions during oxidation at $800^{\circ} \mathrm{C}$

In Fig. 13 the effect of flow rate is investigated by showing the hydrogen production rate at different inlet steam flow rates. The mass flow rate of steam is varied from $3 \mathrm{~g} / \mathrm{min}$ to 1 and 4 $\mathrm{g} / \mathrm{min}$ while the temperature is maintained at $800{ }^{\circ} \mathrm{C}$. Therefore the fluid mixture velocity changes and hence the species have longer travel time in the porous structure, which affects the conversion of steam to hydrogen. The predicted rate via the coupled model shows very good agreement with the measured data. The molar fractions of the fluid mixture inside the reactor, for different inlet flow rates of steam at time $=1 \mathrm{~min}$, are shown in Fig. 14. As expected, the conversion is higher for lower steam flow rates since the fluid particles spend more time within the reactive porous bed, and thus the hydrogen production approaches the thermodynamic limit. However, the overall production rate is lower as it leads to a longer duration of the oxidation step, which usually requires a higher energy input to maintain the reactor temperature, and hence a higher cost of operation.

Fig. 13 Hydrogen production rate at $800^{\circ} \mathrm{C}$ for different inlet steam flow rates.

Fig. 14 Effect of inlet steam flow rates on the mixture molar fractions at $t=1 \mathrm{~min}$

The effect of concentration of steam on the hydrogen production rate is investigated by introducing a controlled stream of helium to the inlet of the reactor and diluting the steam concentration. The fluid particles are then composed of three species, namely steam, hydrogen, and helium. Since the helium does not participate in the chemical reaction, its amount remains constant in each particle. However it changes the bulk density and viscosity. In Fig. 15 the hydrogen production rate is shown for steam inlet molar fractions of $1,0.8,0.6$, and 0.4 . The same inlet flow rate is used to isolate the effect of reactant concentration on the production of hydrogen. The coupled model is capable of predicting the hydrogen production rate, which 
supports the assumption of a first order oxidation reaction. The molar fraction of species inside the porous reactive bed at $\mathrm{t}=1 \mathrm{~min}$ is shown in Fig. 16. Due to the first order nature of the chemical reaction, the conversion of steam to hydrogen decreases significantly by reducing the amount of steam within the inlet flow. Therefore operating the process with a diluted steam flow requires longer time and leads to less energy efficient conversion.

Fig. 15 Effect of inlet steam molar fractions on the hydrogen production rate at $800^{\circ} \mathrm{C}$

Fig. $16 \mathrm{H}_{2} \mathrm{O}$ and $\mathrm{H}_{2}$ molar fractions within the reactive porous bed at $\mathrm{t}=1 \mathrm{~min}$ for different steam inlet molar fractions

\section{Conclusion}

The random walk method has been extended and applied to solve species transport for the water splitting reaction within a reactive porous reactor. The drift velocity component of the random walk equation is modified to recover the species transport equation with variable dispersion and bulk fluid density from the Fokker-Planck equation. The mass generation due to chemical reactions is modeled by changing the mass and composition of the fluid particles. This provides an easy and efficient way to incorporate reactions with fast kinetics. The model is validated against exact solutions for simple advection-diffusion problems. A coupled model consisting of flow, species transport, and chemical reaction modules is introduced for the iron/iron oxide looping process to simulate the mass, momentum and species transport with chemical reaction within porous media. The hydrogen production rate is compared with experimentally measured data, and excellent agreement is observed.

\section{Acknowledgement}

This work was prepared with the support of U.S. Department of Energy under Award numbers DE-FE0001321 and DE-AR0000184.

\section{References}

[1] Holladay J. D., Hu J., King D. L., and Wang Y., 2009, “An overview of hydrogen production technologies," Catalysis Today, 139(4), pp. 244-260.

[2] Baykara S. Z., 2004, "Experimental solar water thermolysis," International Journal of Hydrogen Energy, 29(14), pp. 1459-1469.

[3] Mallouk T. E., 2013, "Water electrolysis: Divide and conquer," Nat Chem, 5(5), pp. 362363.

[4] Nath K., and Das D., 2003, "Hydrogen from biomass," Curr Sci, 85(3), pp. 265-271. 
[5] Turner J. a, 2004, "Sustainable hydrogen production.," Science (New York, N.Y.), 305(5686), pp. 972-4.

[6] Keene D. J., Davidson J. H., and Lipiński W., 2013, “A Model of Transient Heat and Mass Transfer in a Heterogeneous Medium of Ceria Undergoing Nonstoichiometric Reduction," Journal of Heat Transfer, 135(5), p. 052701.

[7] Keene D. J., Lipiński W., and Davidson J. H., 2014, "The effects of morphology on the thermal reduction of nonstoichiometric ceria," Chemical Engineering Science, 111, pp. 231-243.

[8] Steinfeld a, 2002, "Solar hydrogen production via a two-step water-splitting thermochemical cycle based on $\mathrm{Zn} / \mathrm{ZnO}$ redox reactions," International Journal of Hydrogen Energy, 27(6), pp. 611-619.

[9] Steinfeld A., 2005, "Solar thermochemical production of hydrogen-a review," Solar Energy, 78(5), pp. 603-615.

[10] Mehdizadeh A. M., Klausner J. F., Barde A., and Mei R., 2012, "Enhancement of thermochemical hydrogen production using an iron-silica magnetically stabilized porous structure," International Journal of Hydrogen Energy, 37(11), pp. 8954-8963.

[11] Whitaker S., 1998, The method of volume averaging, Springer.

[12] Salamon P., Fernàndez-Garcia D., and Gómez-Hernández J. J., 2006, “A review and numerical assessment of the random walk particle tracking method," Journal of contaminant hydrology, 87(3), pp. 277-305.

[13] Liu G., Zheng C., and Gorelick S. M., 2004, "Limits of applicability of the advectiondispersion model in aquifers containing connected high-conductivity channels," Water Resources Research, 40(8), p. n/a-n/a.

[14] Kinzelbach W., and Uffink G., 1991, "The random walk method and extensions in groundwater modelling," Transport Processes in Porous Media, Springer, pp. 761-787.

[15] Tompson a. F. B., and Dougherty D. E., 1992, "Particle-grid methods for reacting flows in porous media with application to Fisher's equation," Applied Mathematical Modelling, 16(7), pp. 374-383.

[16] LaBolle E. M., Fogg G. E., and Tompson A. F. B., 1996, "Random $\square$ walk simulation of transport in heterogeneous porous media: Local mass $\square$ conservation problem and implementation methods," Water Resources Research, 32(3), pp. 583-593.

[17] Bagtzoglou A. C., Dougherty D. E., and Tompson A. F. B., 1992, “Application of particle methods to reliable identification of groundwater pollution sources," Water Resources Management, 6(1), pp. 15-23. 
[18] Chorin A. J., 1973, “Numerical study of slightly viscous flow,” Journal of Fluid Mechanics, 57(04), pp. 785-796.

[19] Ghoniem a. F., Chorin a. J., and Oppenheim a. K., 1982, "Numerical Modelling of Turbulent Flow in a Combustion Tunnel," Philosophical Transactions of the Royal Society A: Mathematical, Physical and Engineering Sciences, 304(1484), pp. 303-325.

[20] Ghoniem A., and Sherman F., 1985, "Grid-free simulation of diffusion using random walk methods," Journal of Computational Physics, 61(1), pp. 1-37.

[21] Ghoniem a. F., and Oppenheim a. K., 1984, "Numerical solution for the problem of flame propagation by the random element method," AIAA Journal, 22(10), pp. 1429-1435.

[22] Li S., and Liu W. K., 2002, "Meshfree and particle methods and their applications," Applied Mechanics Reviews, 55(1), p. 1.

[23] Bear J., 2013, Dynamics of fluids in porous media, DoverPublications. com.

[24] Wang H., Liang D., Ewing R. E., Lyons S. L., and Qin G., 2003, “An improved numerical simulator for different types of flows in porous media," Numerical Methods for Partial Differential Equations, 19(3), pp. 343-362.

[25] Tompson A. F. B., and Gelhar L. W., 1990, "Numerical simulation of solute transport in three $\square$ dimensional, randomly heterogeneous porous media," Water Resources Research, 26(10), pp. 2541-2562.

[26] Itô K., 1951, “On stochastic differential equations,” Mem. Amer. Math. Soc, 4(4).

[27] Ahlstrom S. W., Foote H. P., Arnett R. C., Cole C. R., and Serne R. J., 1977, Multicomponent mass transport model: Theory and numerical implementation (discreteparcel-random-walk version), Battelle Pacific Northwest Labs., Richland, Wash.(USA).

[28] Prickett T. A., Naymik T. G., and Lonnquist C. G., 1981, A“ random-walk” solute transport model for selected groundwater quality evaluations, Illinois State Water Survey Champaign, IL.

[29] Kinzelbach W., 1988, "The random walk method in pollutant transport simulation," Groundwater flow and quality modelling, Springer, pp. 227-245.

[30] Rao N. J., Borwanker J. D., and Ramkrishna D., 1974, "Numerical solution of Ito integral equations,” SIAM Journal on Control, 12(1), pp. 124-139.

[31] Kloeden P., and Pearson R., 1977, "The numerical solution of stochastic differential equations," The Journal of the Australian ..., 20(September 1976), pp. 8-12. 
[32] Kinzelbach W., 1987, "Methods for the simulation of pollutant transport in ground water-A model comparison," Proc. Conf. on Solving Ground Water Problems with Models, Denver, Colorado, pp. 656-674.

[33] Mehdizadeh A. M., Klausner J. F., Barde A., Rahmatian N., and Mei R., 2012, "Investigation of hydrogen production reaction kinetics for an iron-silica magnetically stabilized porous structure," International Journal of Hydrogen Energy, 37(18), pp. 13263-13271.

[34] Kumar A., Jaiswal D. K., and Kumar N., 2009, “Analytical solutions of one-dimensional advection-diffusion equation with variable coefficients in a finite domain," Journal of Earth System Science, 118(5), pp. 539-549.

[35] Van Genuchten M. T., and Alves W. J., 1982, "Analytical solutions of the onedimensional convective-dispersive solute transport equation," United States. Dept. of Agriculture. Technical bulletin. 
Table 1. List of validation tests

\begin{tabular}{|c|c|c|c|c|c|c|}
\hline & & $\begin{array}{c}\text { Compared } \\
\text { with }\end{array}$ & $\langle u\rangle$ & $D$ & $\langle\rho\rangle^{F}$ & $\left\langle\dot{r}_{j}\right\rangle$ \\
\hline 1 & $\begin{array}{l}\text { AD with constant } \\
\text { properties }\end{array}$ & $\begin{array}{c}\text { Exact } \\
\text { solution }\end{array}$ & Constant & Constant & Constant & Zero \\
\hline 2 & $\begin{array}{c}\text { AD with variable } \\
\text { density }\end{array}$ & FD solution & Constant & Constant & Varying & Zero \\
\hline 3 & $\begin{array}{l}\text { AD with variable } \\
\text { velocity and } \\
\text { dispersion }\end{array}$ & $\begin{array}{c}\text { Exact } \\
\text { solution }\end{array}$ & $\begin{array}{l}\text { Linearly } \\
\text { varying }\end{array}$ & $\begin{array}{l}\text { Quadratic } \\
\text { Variation }\end{array}$ & Constant & Zero \\
\hline 4 & AD with generation & $\begin{array}{c}\text { Exact } \\
\text { solution }\end{array}$ & Constant & Constant & Constant & $\begin{array}{c}\text { Constant/Firs } \\
\text { t order }\end{array}$ \\
\hline
\end{tabular}


Figure 1

Click here to download high resolution image

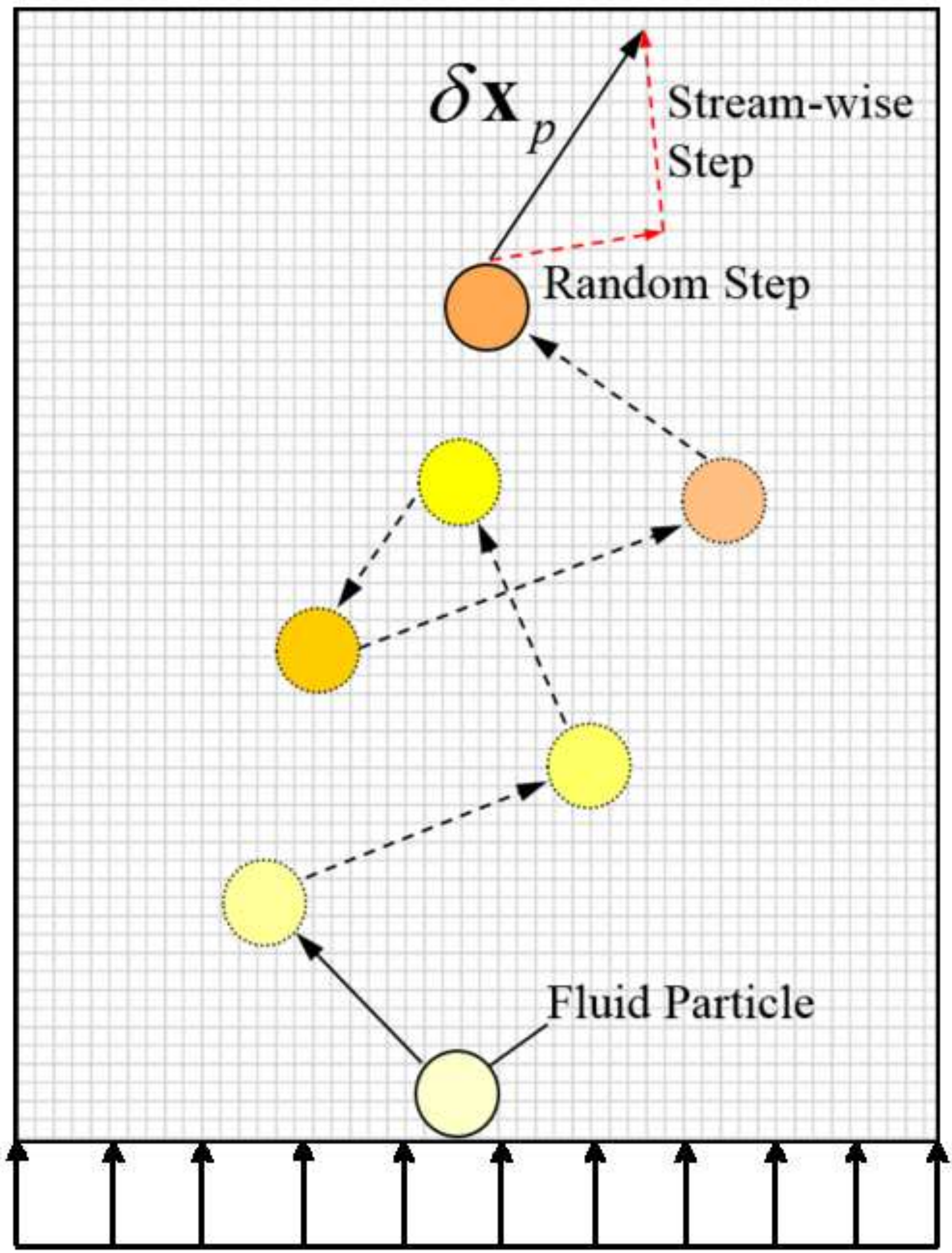

Flow Direction 


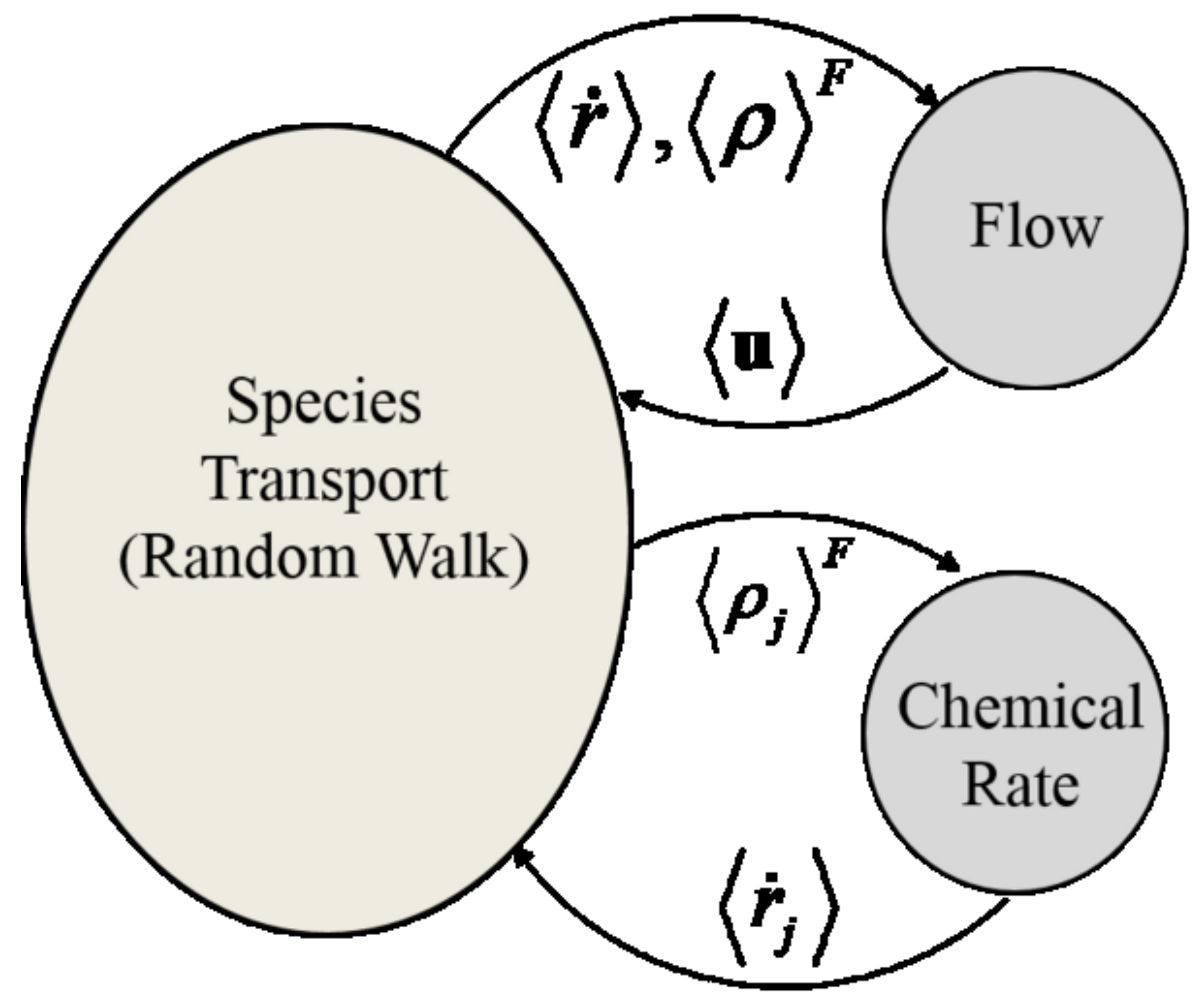


Click here to download high resolution image

\section{Porous Medium}

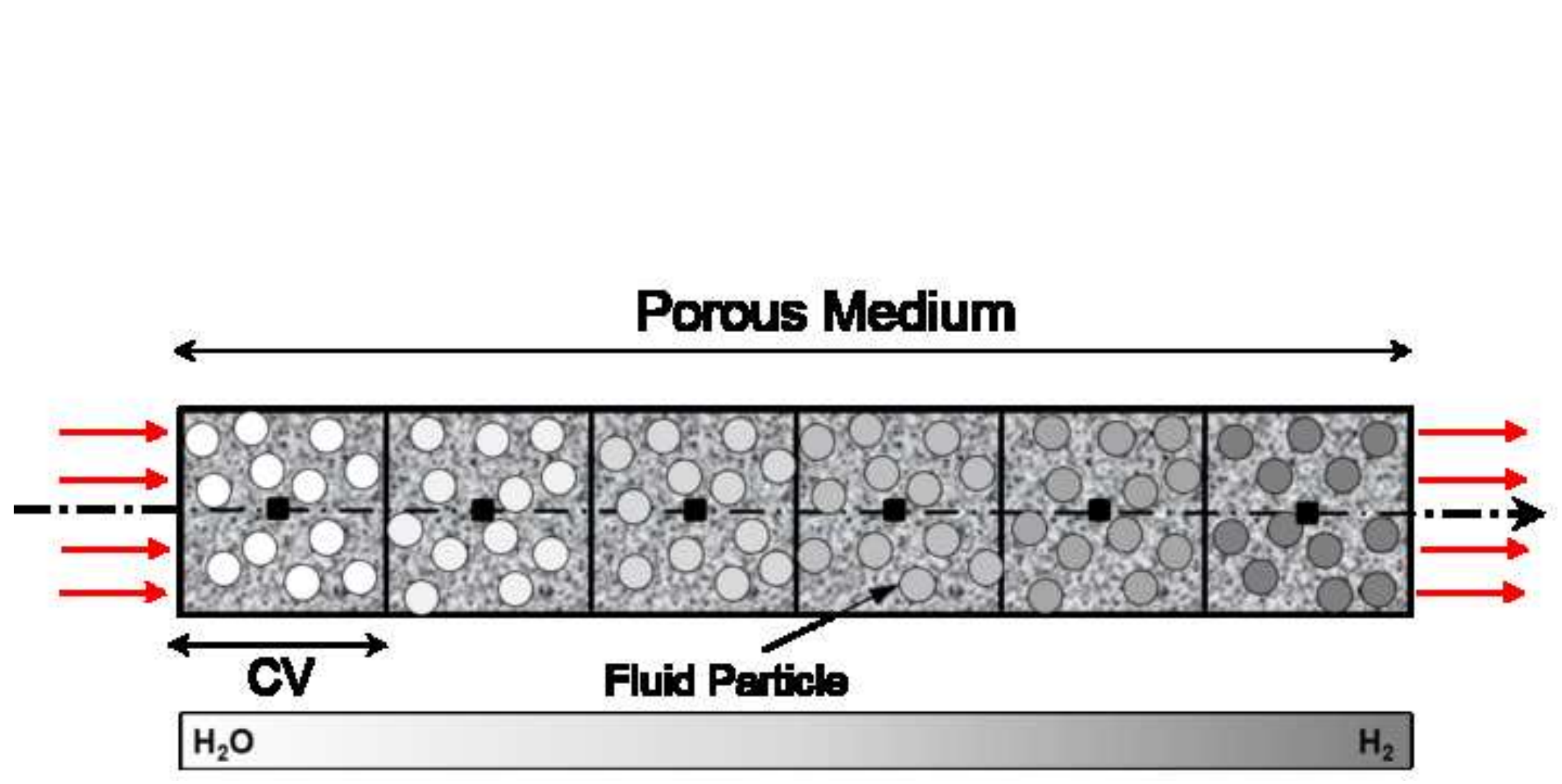




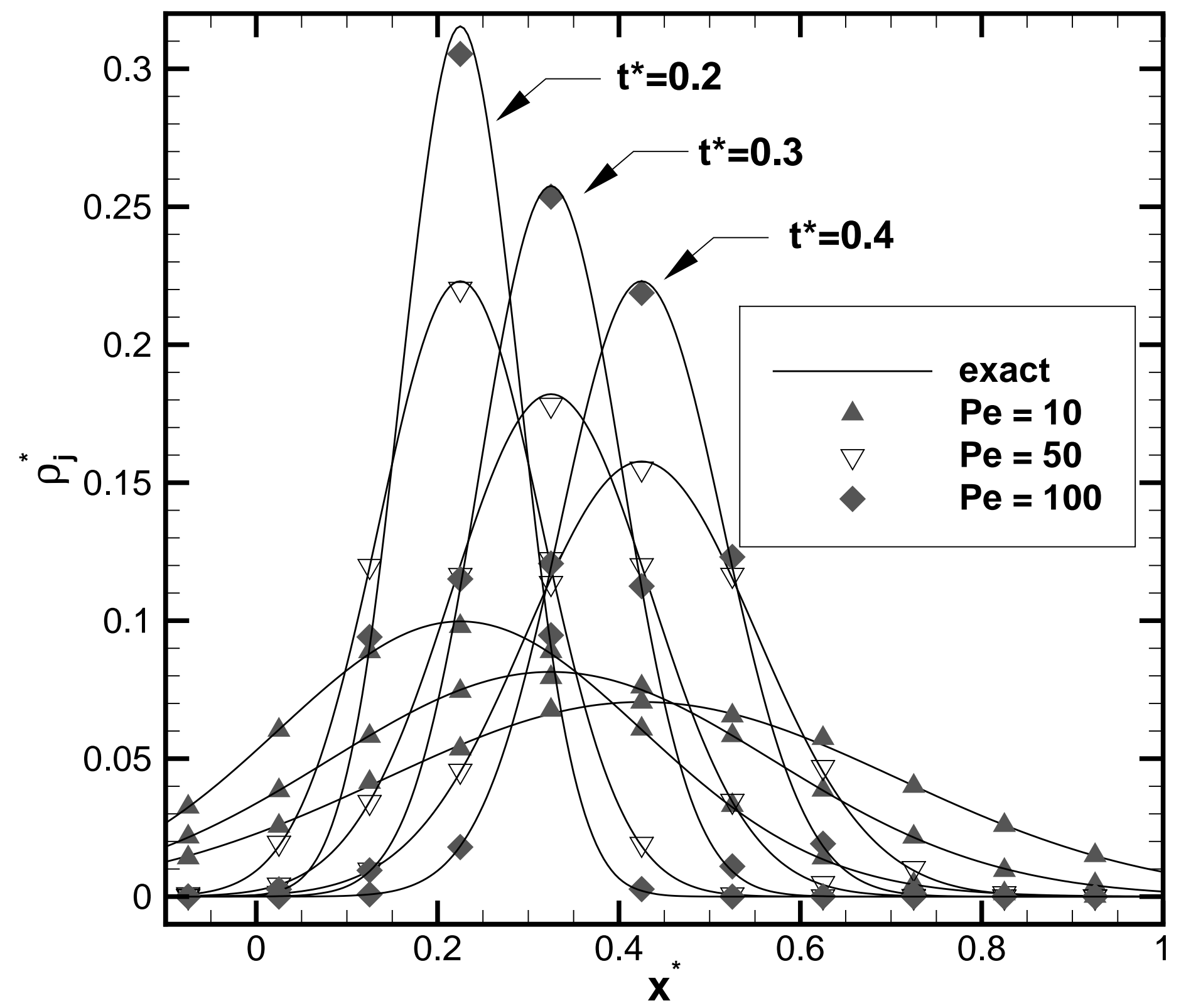




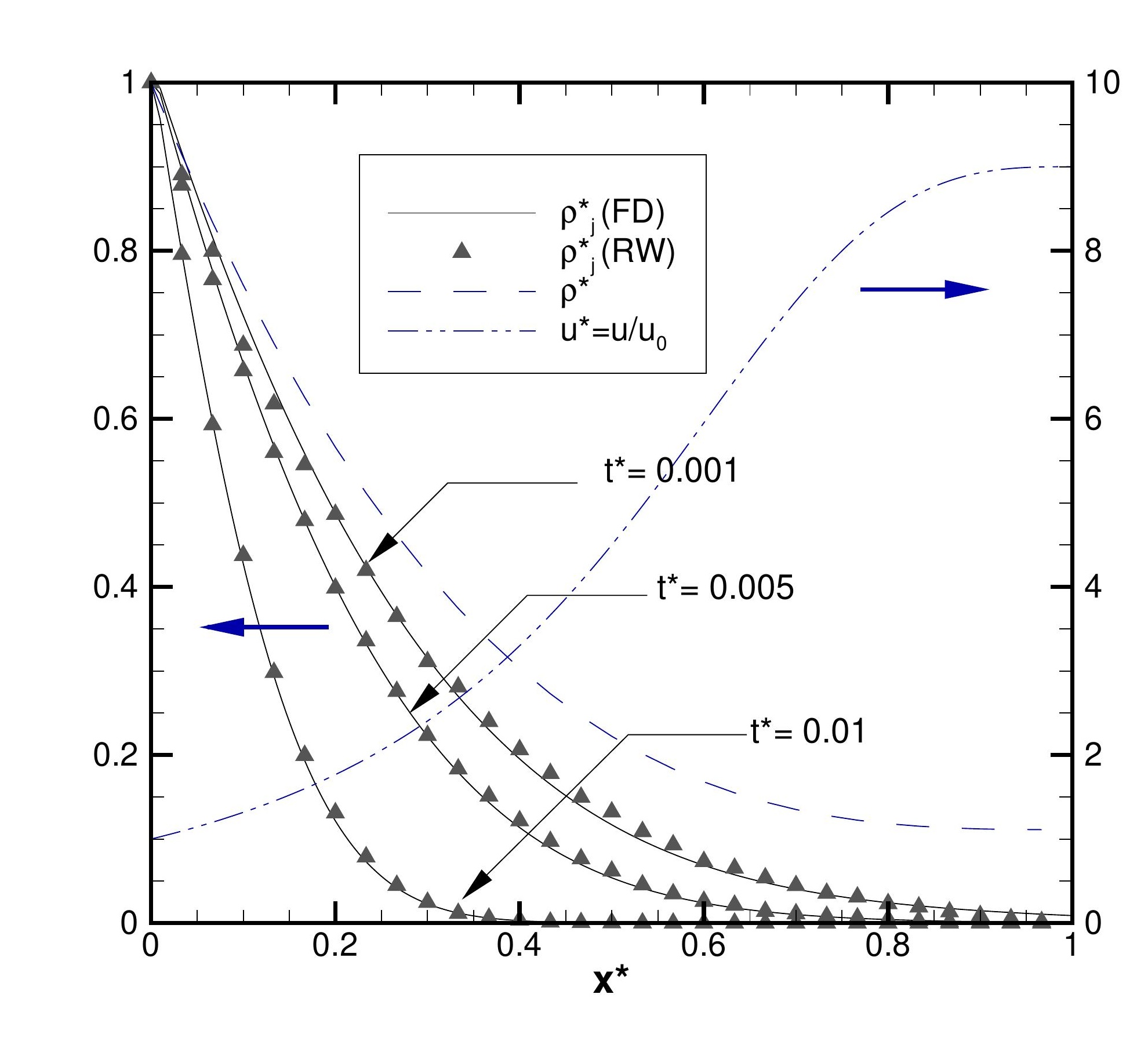

Figure 5

10

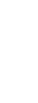

\section{Figure 5}

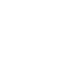
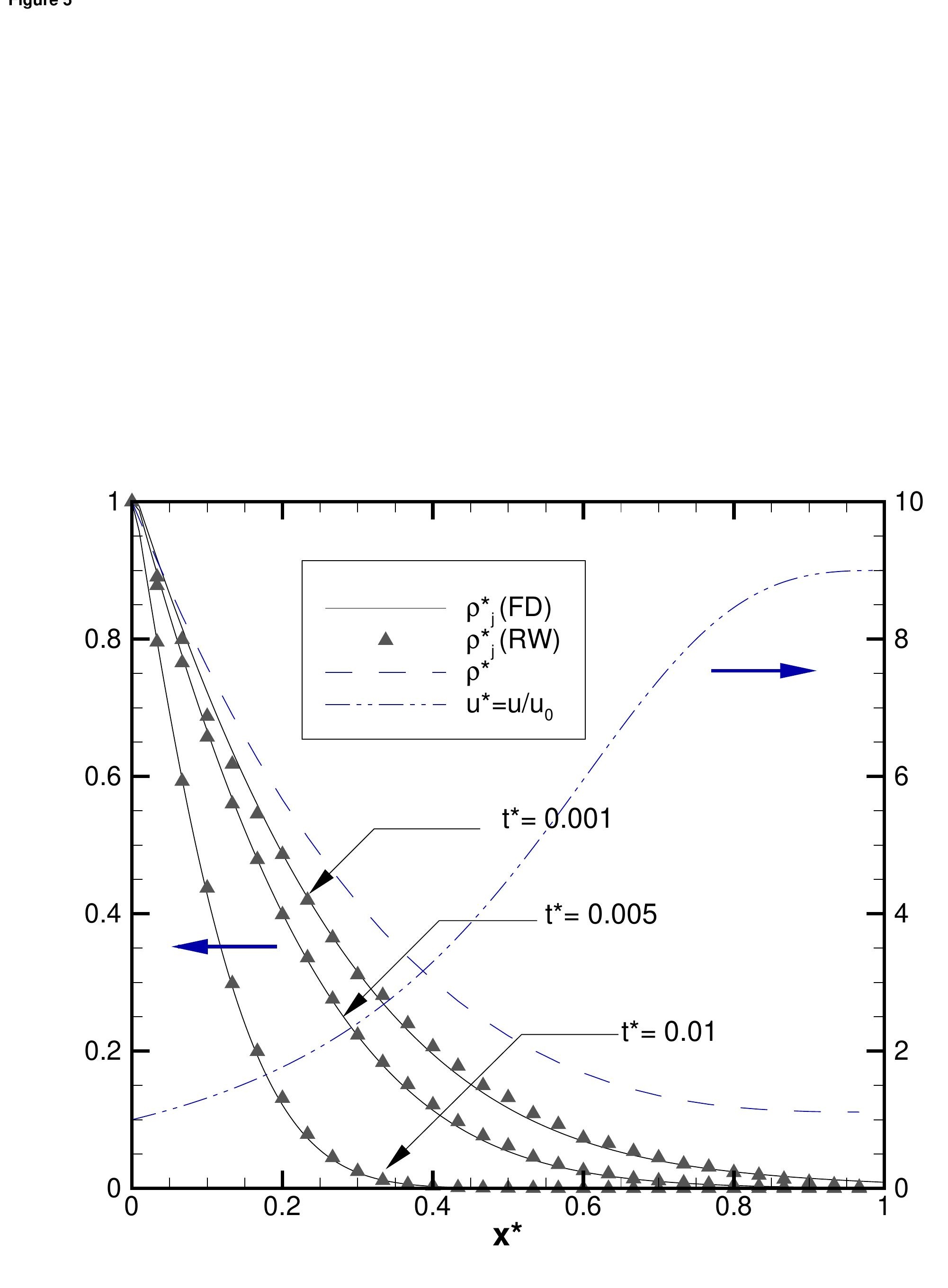


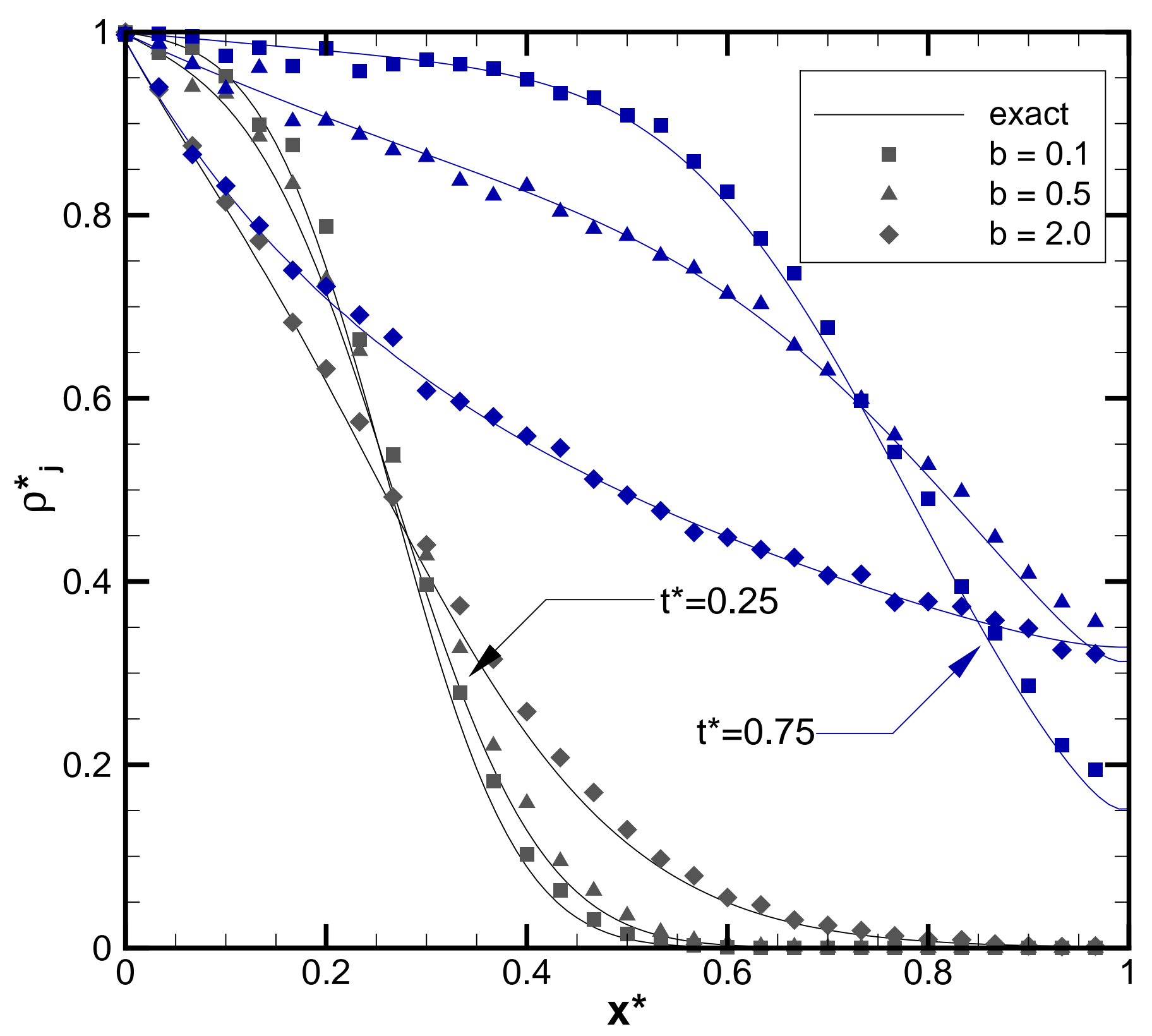

Figure 6 


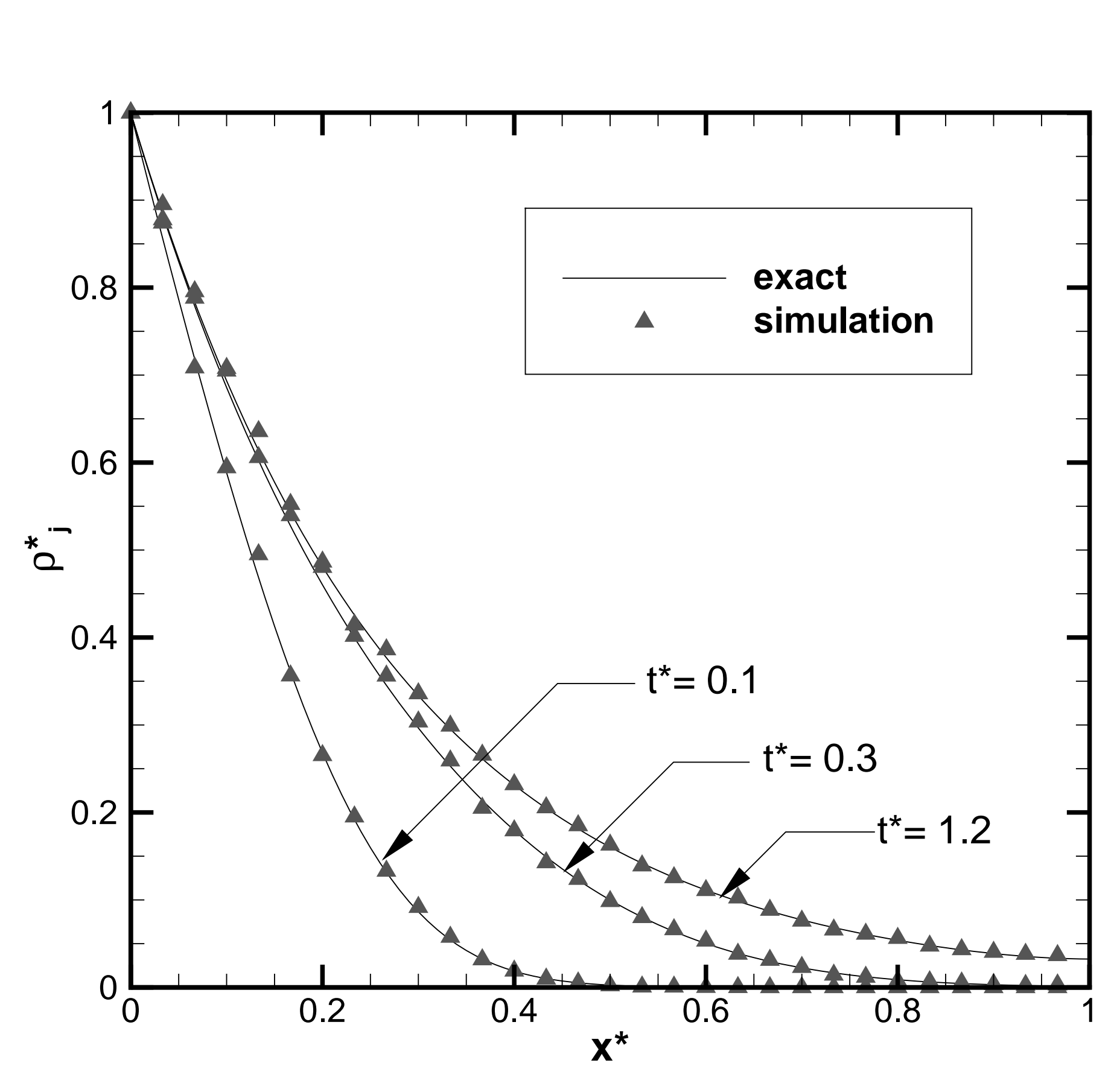

Figure 8

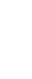

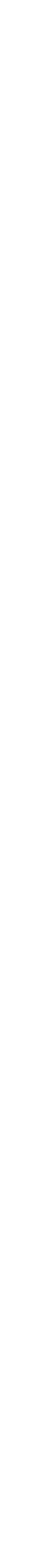

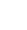

Figure

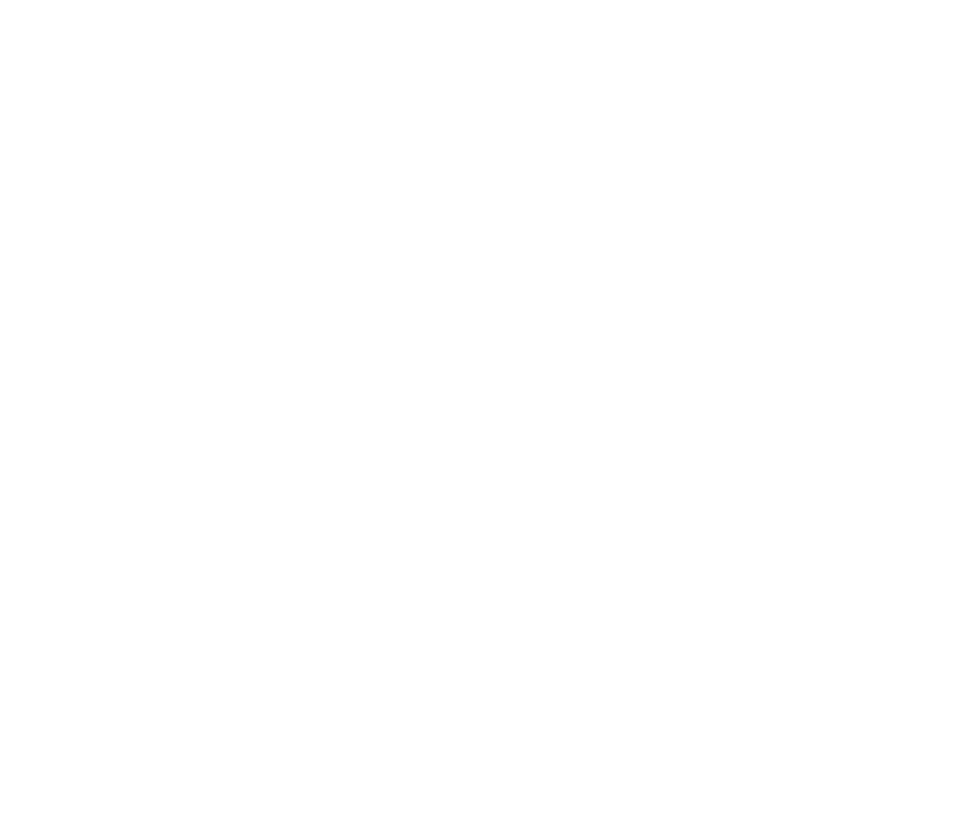


Figure 9

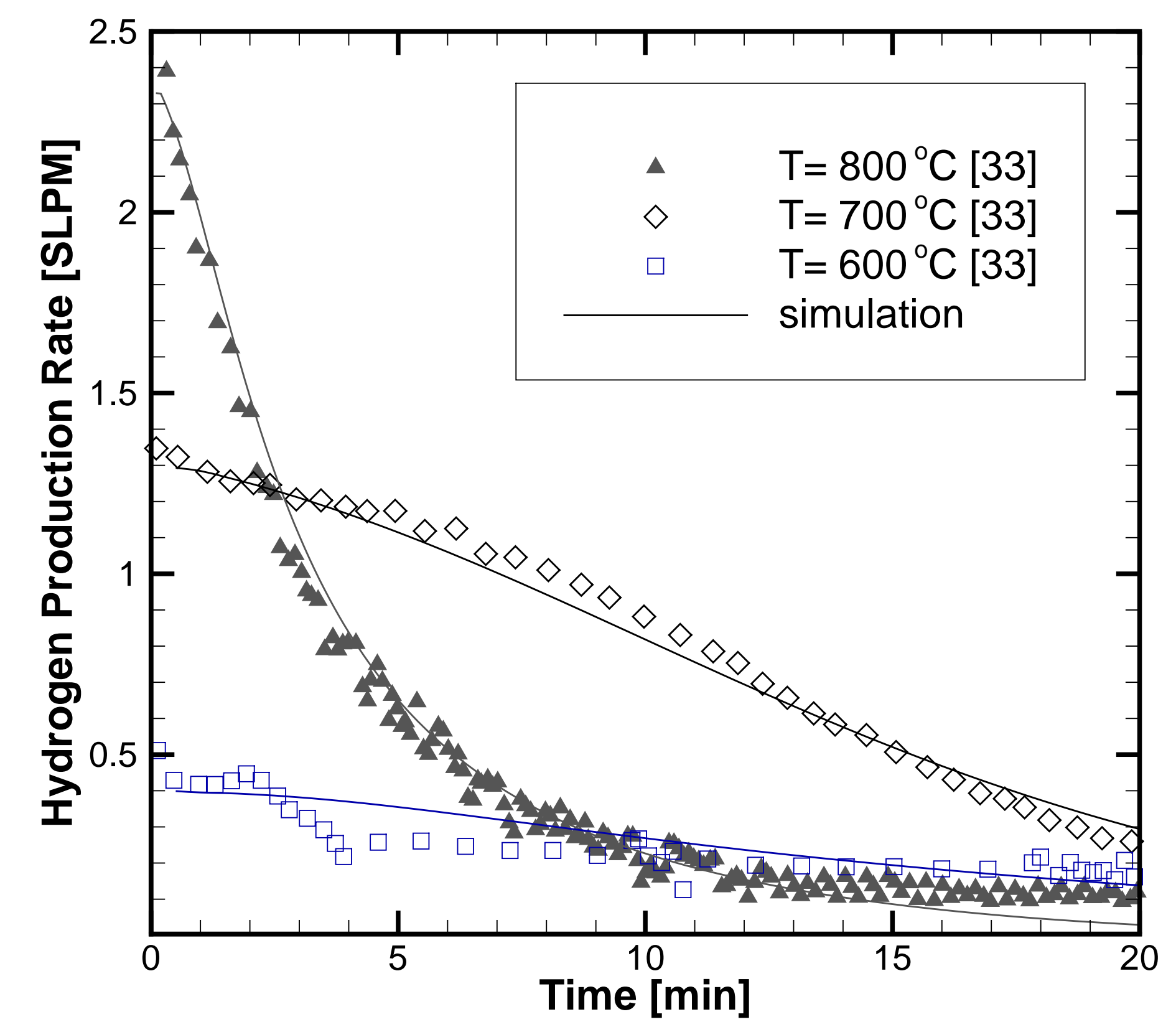




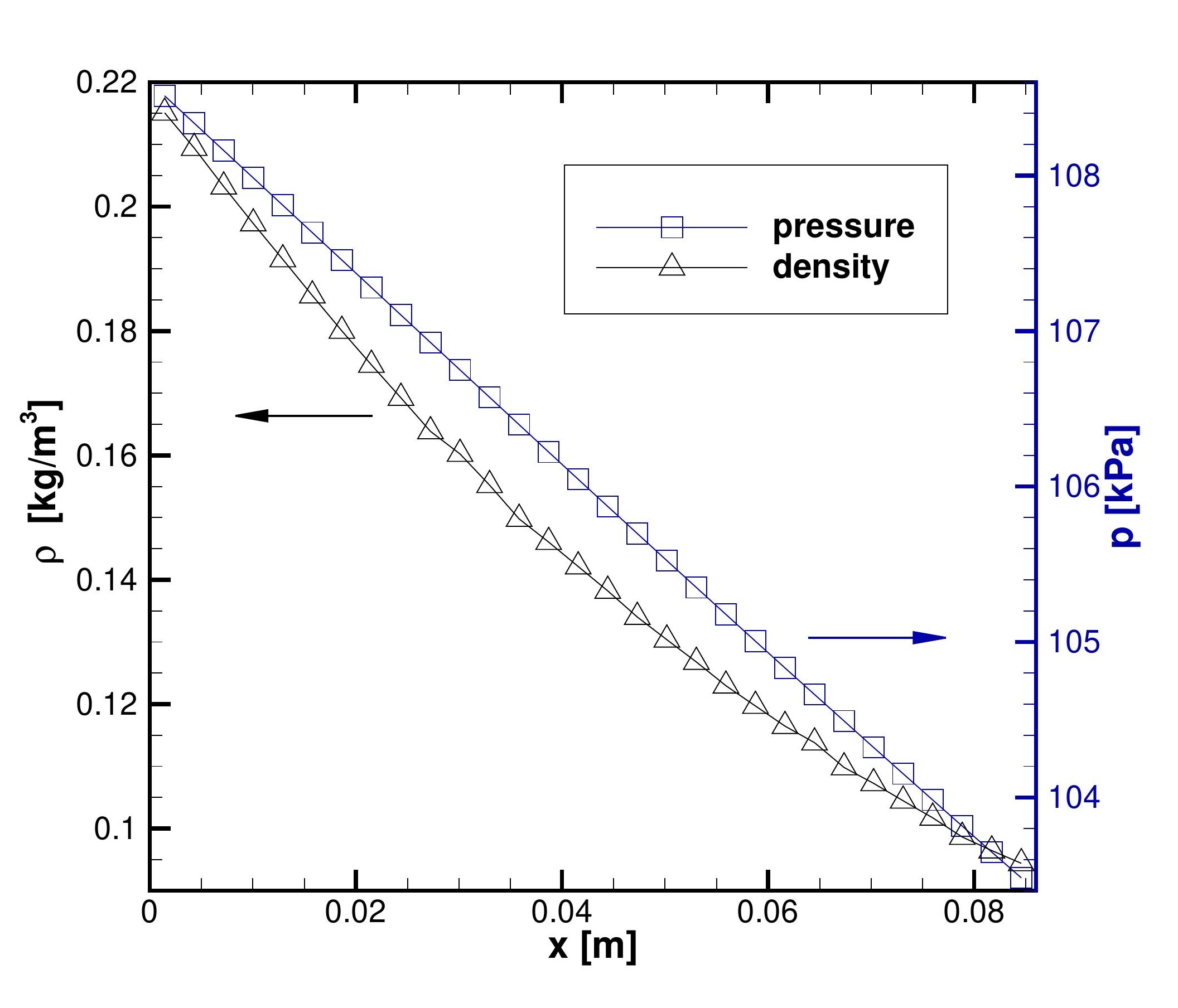

Figure 10

Figure 10

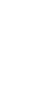
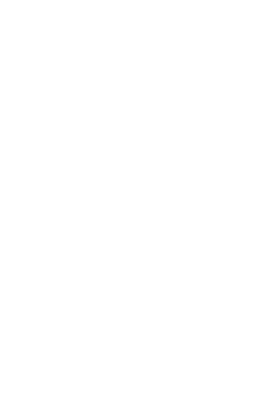


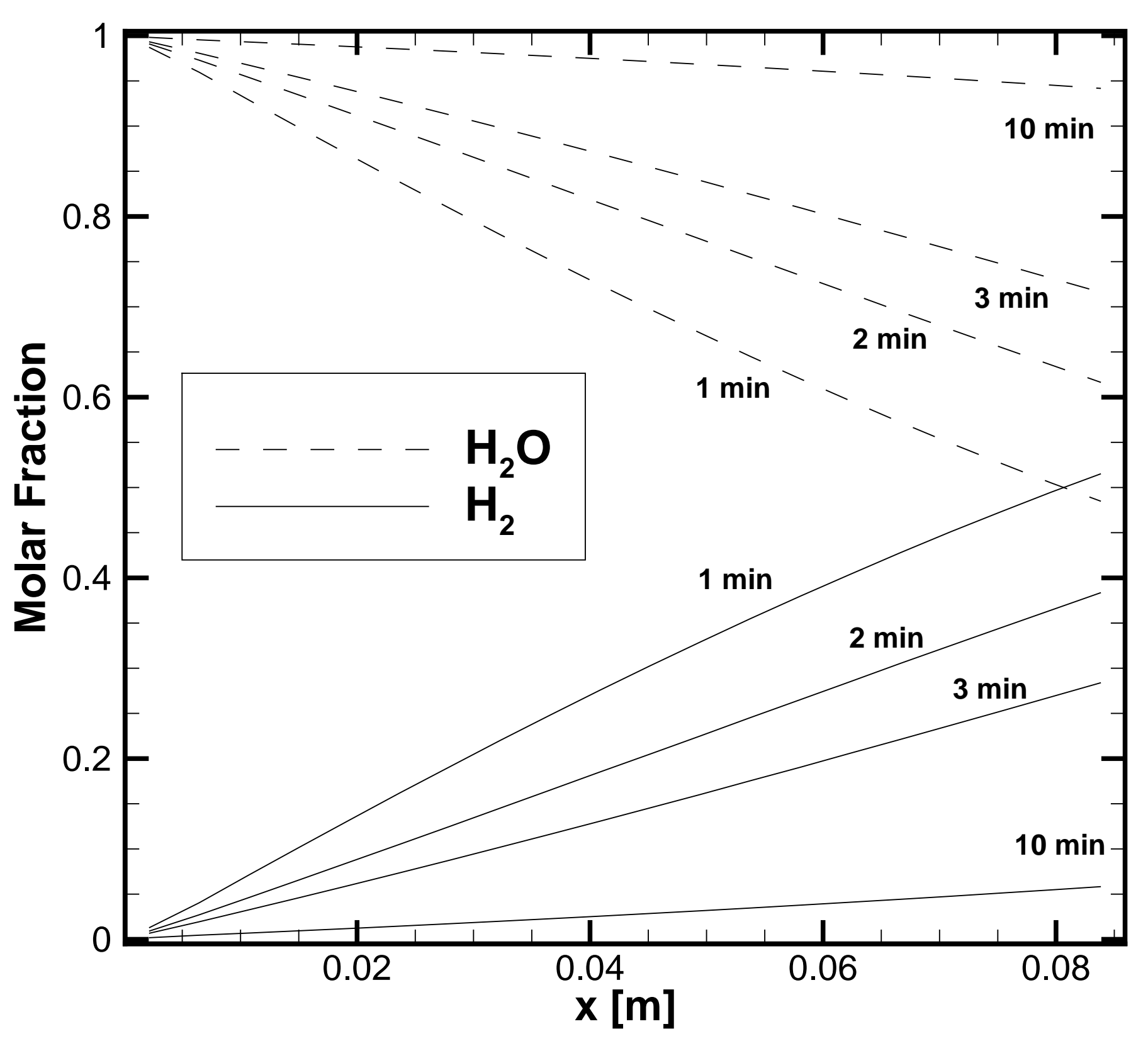

Figure 12 


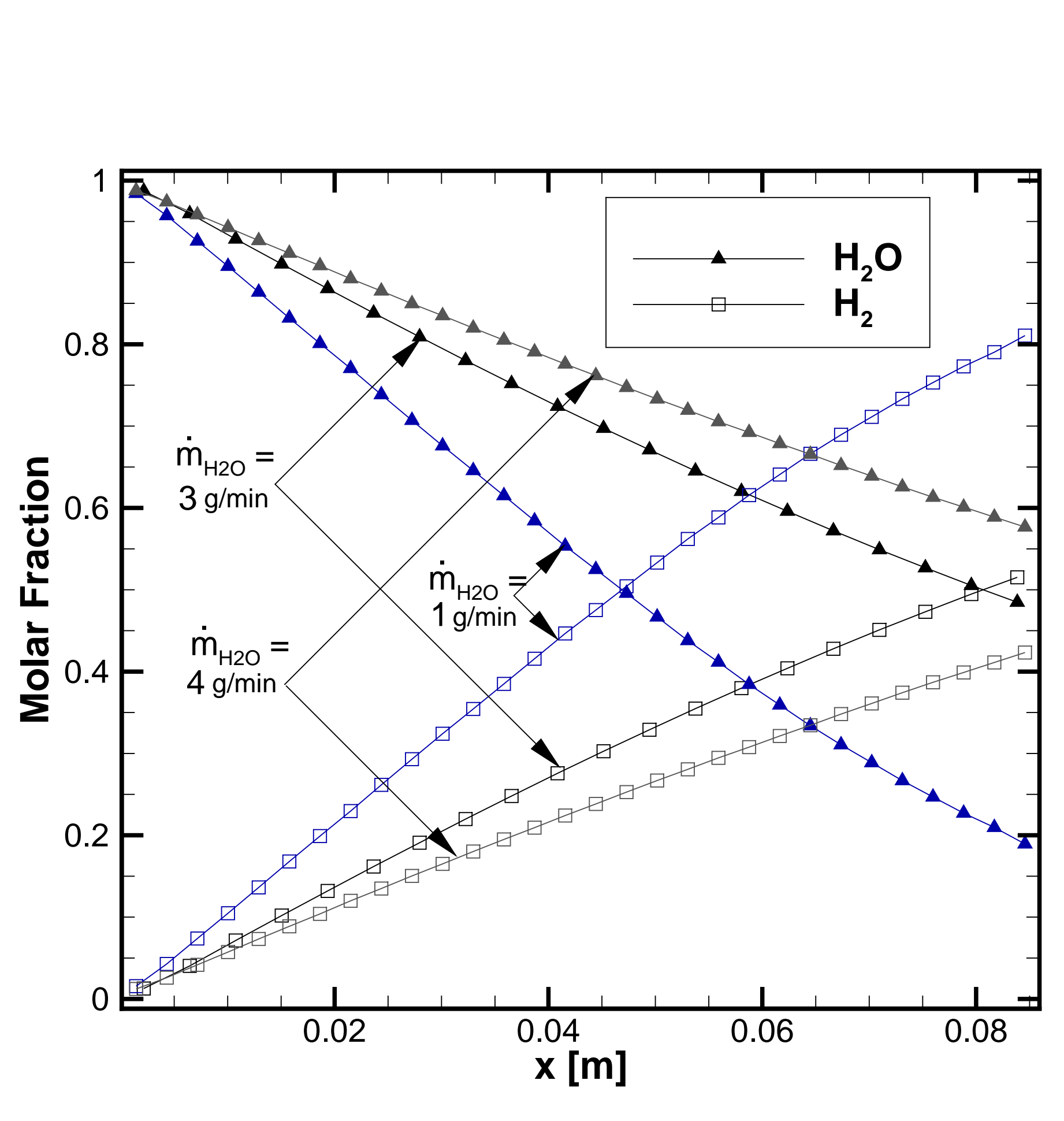

Figure 14

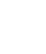

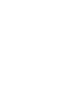
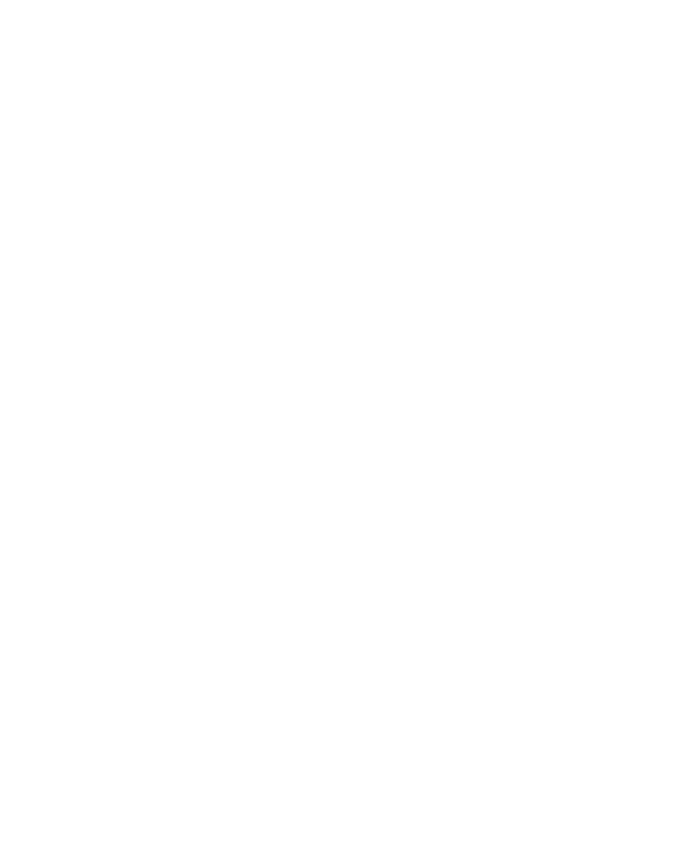

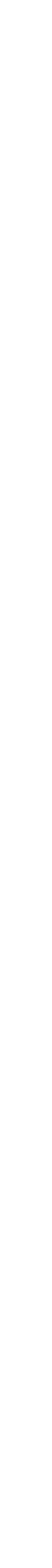

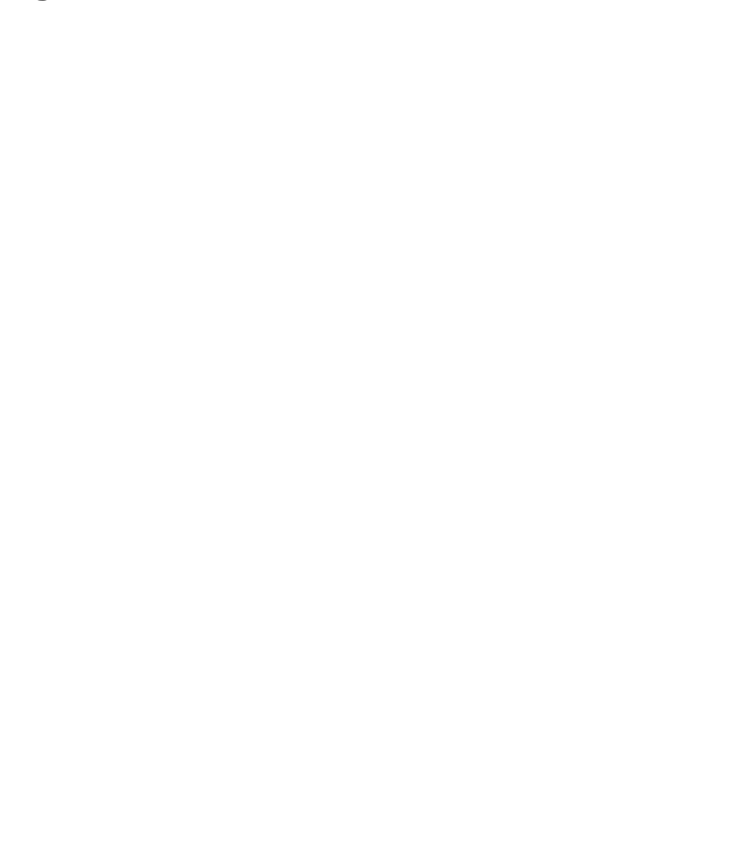




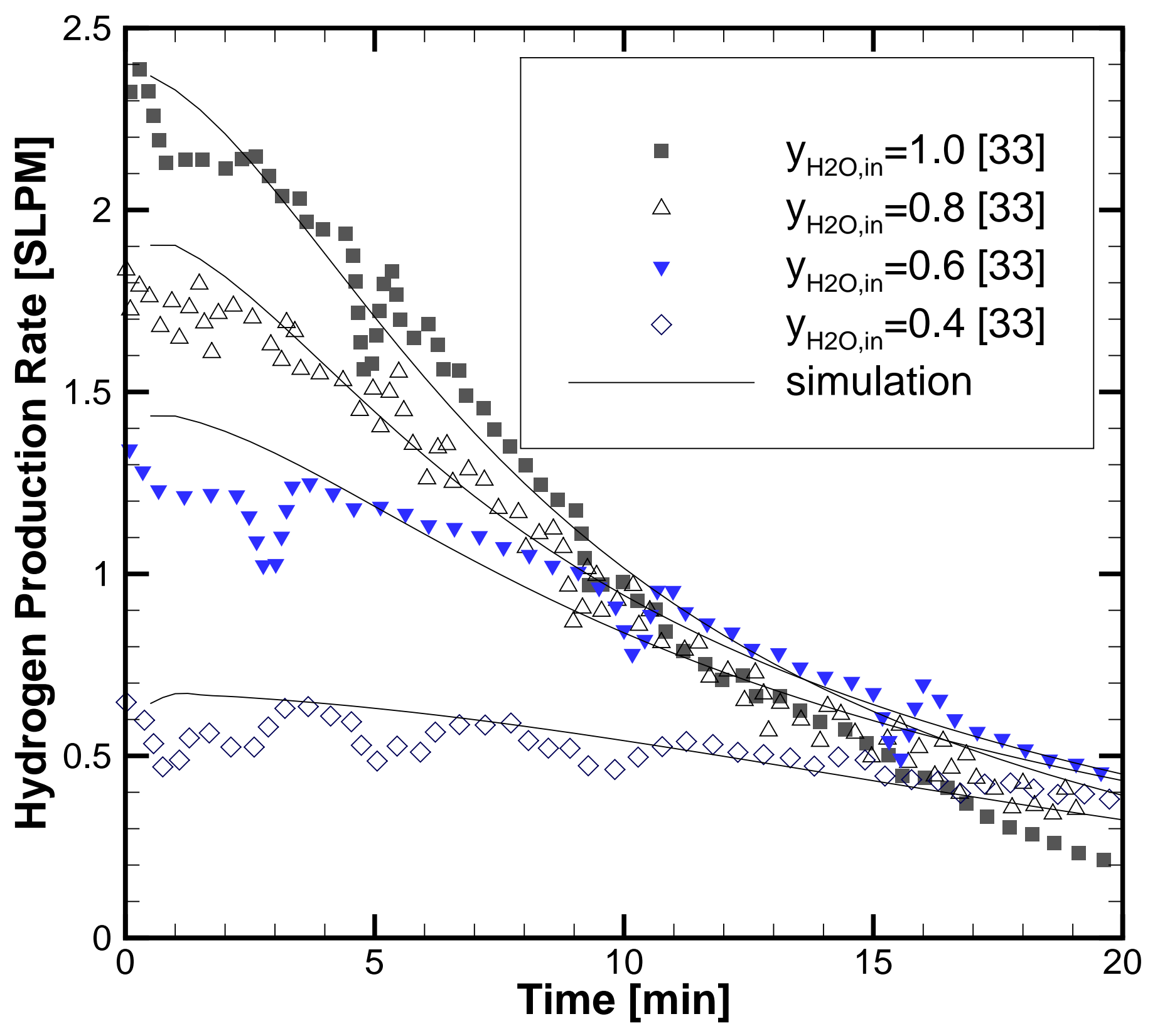




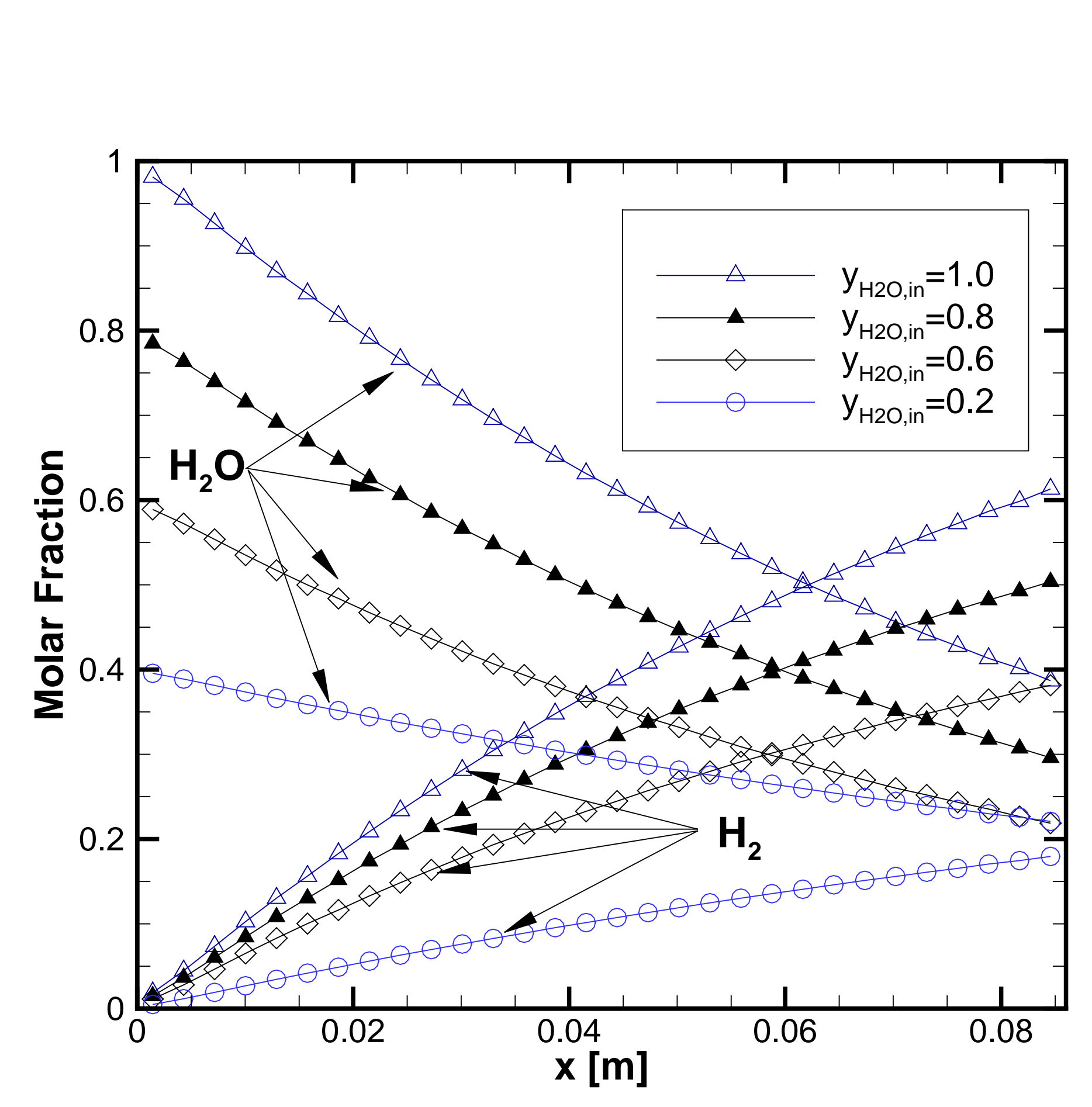

Figure 16

\section{Figure 16}

.

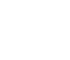

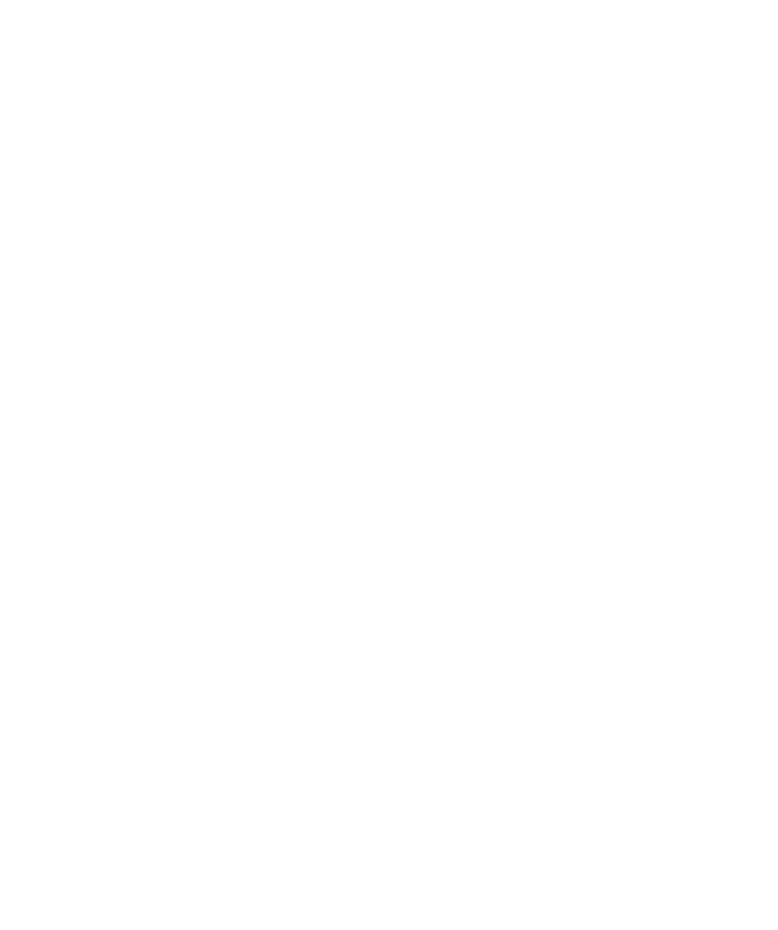

